\title{
A simplified method for calculating spectral emission of nonequilibrium air plasmas in hypersonic shock-layers
}

\author{
Xin $\mathrm{He}^{1}$, Cheng Gao ${ }^{1}$ and Tao Jiang ${ }^{2^{*}}$
}

* Correspondence:
fengqiaoren999@163.com
${ }^{2}$ Computational Aerodynamics
Institute, China Aerodynamics
Research and Development Center,
Mianyang 621000, China
Full list of author information is
available at the end of the article

available at the end of the article

\begin{abstract}
A simplified method for calculating the spectral emission of nonequilibrium air plasmas is developed. In order to obtain the nonequilibrium energy level populations, the nonequilibrium coefficients are introduced into the Saha-Boltzmann equation. These nonequilibrium coefficients are calculated by using several significant radiative processes. An approach to the determination of nonequilibrium electronic energy level populations of diatomic molecules is also presented. Based on the method, spectral emission of atoms and molecules in a typical air plasma cell is investigated. The results reveal that there is a significant difference between the nonequilibrium and equilibrium emission. We apply the method to the nonequilibrium AVCO R-156 experiment. Good agreement with the NEQAIR code and the measured data is shown, indicating that the method is reasonable and has good accuracy.

Keywords: Spectral emission, Nonequilibrium, Air plasma, Hypersonic
\end{abstract}

\section{Springer Open}

\section{Introduction}

During the hypersonic reentry, a significant portion of the heating experienced by a vehicle may be due to the emission of air plasmas in the shock-layer. To investigate the radiative heating, it is highly important to calculate the spectral emission of air plasmas [1].

The air plasmas in the shock-layer may be equilibrium or nonequilibrium [2, 3]. For equilibrium air plasmas, their spectral emission can be easily calculated by means of equilibrium statistical and spectral theory. However, for nonequilibrium air plasmas, the prediction of their emission is a challenge.

For this reason, most of the physical models and computational codes in the literature have tried their best to address nonequilibrium radiation [4-10]. Full models, such as the collisional-radiative (CR) model, are well-known to be accurate [11, 12]. However, their computational cost is very high. To improve the computational efficiency, researchers tend to develop simplified models. These simplified models will be desirable and useful in engineering. The nonequilibrium air radiation (NEQAIR) code

(c) The Author(s). 2021 Open Access This article is licensed under a Creative Commons Attribution 4.0 International License, which permits use, sharing, adaptation, distribution and reproduction in any medium or format, as long as you give appropriate credit to the original author(s) and the source, provide a link to the Creative Commons licence, and indicate if changes were made. The images or other third party material in this article are included in the article's Creative Commons licence, unless indicated otherwise in a credit line to the material. If material is not included in the article's Creative Commons licence and your intended use is not permitted by statutory regulation or exceeds the permitted use, you will need to obtain permission directly from the copyright holder. To view a copy of this licence, visit http://creativecommons.org/licenses/by/4.0/. 
presented by Park is the most widely used. Although NEQAIR employed the so-called quasi-steady-state (QSS) assumption [13, 14], its computational cost is not low due to the consideration of many collisional and radiative processes in air plasmas [15].

In this paper, based on the main idea of the CR model, a simplified method for calculating nonequilibrium air plasma radiation is developed. The method uses a modified Saha-Boltzmann equation to represent the nonequilibrium energy level population. By solving the conservation equation containing significant radiative processes, the nonequilibrium population of bound electronic energy levels can be obtained. Based on a typical air plasma cell, the difference between the nonequilibrium and equilibrium emission of particles is analyzed. In addition, application of the method in nonequilibrium AVCO R-156 experiments is discussed.

\section{Simplified method}

In the following, we consider the 11-species air model that contains $\mathrm{N}, \mathrm{N}^{+}, \mathrm{O}, \mathrm{O}^{+}, \mathrm{N}_{2}$, $\mathrm{N}_{2}^{+}, \mathrm{O}_{2}, \mathrm{O}_{2}^{+}, \mathrm{NO}, \mathrm{NO}^{+}$and free electrons. The method for determining the bound electronic energy level populations is first explained, which is based on our previous work $[16,17]$. Then, we apply the method to some typical plasmas and analyze the nonequilibrium radiation from atoms and molecules, respectively.

\subsection{Bound electronic energy level populations}

The main idea of the full CR model is that the population of each bound electronic energy level is affected by various collisional and radiative processes [1]. If the QSS assumption is used, the population of the $i$ th bound electronic energy level $n_{i}$ is determined by

$$
\left.\frac{d n_{i}}{d t}\right|_{\text {collisional }}+\left.\frac{d n_{i}}{d t}\right|_{\text {radiative }}=0
$$

In Eq. (1), the collisional processes can be subdivided into cases where the impacting particles are electrons, ions, or neutral particles. The radiative processes include radiative transition, radiative recombination, and so on $[1,12]$. The full CR model is accurate because it considers as many processes as possible. However, this leads to a large number of coupled rate-equations and a huge increase in the computational cost.

For general hypersonic air plasmas, the departure from thermal equilibrium is usually not very large. By introducing a nonequilibrium coefficient into the Saha-Boltzmann equation $[16,17]$, there will be

$$
n_{i}=b_{i} n_{e} n^{+} \frac{g_{i}}{2 Q^{+}}\left(\frac{h^{2}}{2 \pi m_{e} k T_{e}}\right)^{3 / 2} \exp \left(\frac{I_{i}}{k T_{e}}\right)
$$

where $b_{i}$ is the nonequilibrium coefficient for the population of the $i$ th level, $n_{e}$ and $n^{+}$ are the number density of free electrons and charged particles, $Q^{+}$is the partition sum of charged particles, $g_{i}$ and $I_{i}$ are the degeneracy and ionization energy of the $i$ th level, $T_{e}$ is electronic temperature, $m_{e}$ is electron mass, $h$ is Planck constant, and $k$ is Boltzmann constant.

To calculate the $b_{i}$ in Eq. (2), three most significant radiative processes, that is, radiative recombination, spontaneous transitions and photo excitation from the ground state, are considered. After some simplifications are made [16], this leads to 


$$
n_{i} \sum_{j=2}^{i-1} A_{i j} \approx \sum_{j=i+1}^{\infty} n_{j} A_{j i}+n_{e} n_{0}^{+} R_{i}
$$

where $n_{j}$ is the population of the $j$ th level, $A_{i j}$ and $A_{j i}$ are the transition probabilities, $n_{0}^{+}$is the number density of ground-state charged particles, and $R_{i}$ is the radiative recombination coefficient of the $i$ th level. $R_{i}$ has the form [1]:

$$
R_{i}=5.20 \times 10^{-14} p \sqrt{\frac{I_{i}}{I_{H}}}\left(\frac{I_{i}}{k T_{e}}\right)^{3 / 2} e^{\frac{I_{i}}{k_{e}}} E_{1}\left(\frac{I_{i}}{k T_{e}}\right) G_{p}
$$

where $p$ is the primary quantum number of the $i$ th level, $I_{H}$ is the ionization energy of atomic hydrogen, and $G_{p}$ the Gaunt coefficient ( $G_{p}=1.0$ in the following calculations).

Based on Eqs. (2) and (3), $b_{i}$ can be calculated, and then the bound electronic energy level populations are obtained.

Specifically, the ionization in nonequilibrium air plasmas is usually highly insufficient [18]. Thus, for several lowest levels, the Boltzmann distribution characterized by $T_{e}$ can be directly assumed [19], as NEQAIR code did. For several highest levels, the Saha distribution characterized by $T_{e}$ can be used [17]. For the other levels, the population should be calculated by Eqs. (2) and (3).

\subsection{Atomic emission}

When the speed of a vehicle is high in earth reentry, the diatomic species behind the shock may be highly dissociated [20]. In this situation, spectral emission of $\mathrm{N}$ and $\mathrm{O}$ may be dominant [21, 22]. Therefore, calculation of atomic radiation is highly important.

We employ Park's electronic level system [2]. The nonequilibrium air plasmas is typically optically thick for radiation below $200 \mathrm{~nm}$. It means that the atomic lines corresponding to the transitions from high levels to levels 1-3 can be totally absorbed. Thus, in Eq. (3), the ground state(s) is not level 1 but are levels 1-3. Eq. (3) transforms to

$$
n_{i} \sum_{j=4}^{i-1} A_{i j}=\sum_{j=i+1}^{j_{\max }} n_{j} A_{j i}+n_{e} n_{1}^{+} R_{i}
$$

where $j_{\max }$ equals to 22 (for $\mathrm{N}$ ) or 19 (for O).

To improve the efficiency, it is not necessary to calculate the nonequilibrium population of all levels. Table 1 lists the atomic lines based on NEQAIR [7]. As mentioned above, atomic lines below $200 \mathrm{~nm}$ are almost totally absorbed. For the prediction of radiative heating, the atomic lines above $200 \mathrm{~nm}$ are of great importance. Most of the strong atomic lines above $200 \mathrm{~nm}$ are related to levels 6-12. For these levels, we need surely to calculate their nonequilibrium populations according to Eqs. (2) and (3). For

Table 1 Atomic lines analysis

\begin{tabular}{llll}
\hline atoms & total lines & above $\mathbf{2 0 0} \mathbf{~ n m}$ & strong and related to levels $\mathbf{6 - 1 2}$ \\
\hline $\mathrm{N}$ & 179 & 107 & 87 \\
$\mathrm{O}$ & 109 & 83 & 40 \\
\hline
\end{tabular}


other lower or higher levels, the use of the equilibrium population would lead to small errors in the spectral emission calculation.

To investigate the difference between the nonequilibrium and equilibrium radiation of atoms, we chose an arbitrary air plasma cell. Table 2 lists the condition of the air plasma cell [9]. In the spectral calculations, we only consider bound-bound transitions of $\mathrm{N}$ and $\mathrm{O}$. For the shape of each emission line, the Voigt profile is assumed [3].

Figure 1 shows spectral emission of the two atomic species. In Figs. 1(a) and (b), the electronic energy level population of $\mathrm{N}$ and $\mathrm{O}$ is assumed to be Boltzmannian with $T_{e}$. However, in Figs. 1(c) and (d), the population of $\mathrm{N}$ and $\mathrm{O}$ is calculated by means of the above-mentioned method. One can immediately find that the characteristics of the spectrum are significantly different. For the emission of N, we draw a comparison between Figs. 1(a) and (c). It is shown that the Boltzmannian assumption will cause the emission to be overestimated. For $\mathrm{O}$, a similar phenomenon is seen by a comparison between Figs. 1(b) and (d). The results imply that the use of Boltzmannian distribution could lead to a considerable error in the prediction of radiative heating.

\subsection{Diatomic molecular emission}

For diatomic molecules in air plasmas, the relaxation of electronic energy levels is quicker than vibrational levels and dissociation processes [23]. In order to make use of Eq. (3), we need to determine the radiative recombination coefficient.

We first attempt to found a relationship between the primary quantum number and the ionization energy of a certain electronic level. If the Hydrogen-like approximation is used, this relationship will be

$$
\omega_{i}=\left(I_{H} / I_{i}\right)^{1 / 2}
$$

where $\omega_{i}$ is the primary quantum number of the $i$ th level of molecules. Considering the important electronic energy levels of diatomic molecules for air plasma radiation, we can obtain Table 3 for the three diatomic molecules [24, 25].

Assuming that the electronic, vibrational and rotational energy modes of diatomic molecules are independent, the energy of the $i$ th level can be expressed as [13].

$$
E(e, v, J)=E(e)+G(v)+F(J)
$$

Table 2 The condition of the air plasma cell

\begin{tabular}{ll}
\hline Parameters & Value \\
\hline translational temperature $T_{t,} \mathrm{~K}$ & 31,113 \\
rotational temperature $T_{r}, \mathrm{~K}$ & 18,027 \\
vibrational temperature $T_{v,} \mathrm{~K}$ & 12,280 \\
electronic temperature $T_{e}, \mathrm{~K}$ & 14,888 \\
$\mathrm{~N}_{1} \mathrm{~cm}^{-3}$ & $1.34 \times 10^{16}$ \\
$\mathrm{O}_{1} \mathrm{~cm}^{-3}$ & $1.01 \times 10^{17}$ \\
$\mathrm{~N}_{2}^{+}, \mathrm{cm}^{-3}$ & $2.27 \times 10^{16}$ \\
$\mathrm{~N}_{2}, \mathrm{~cm}^{-3}$ & $1.75 \times 10^{19}$ \\
$\mathrm{~N}^{+}, \mathrm{cm}^{-3}$ & $1.59 \times 10^{14}$ \\
$\mathrm{O}^{+}, \mathrm{cm}^{-3}$ & $1.24 \times 10^{14}$ \\
free electrons, $\mathrm{cm}^{-3}$ & $5.02 \times 10^{14}$ \\
\hline
\end{tabular}




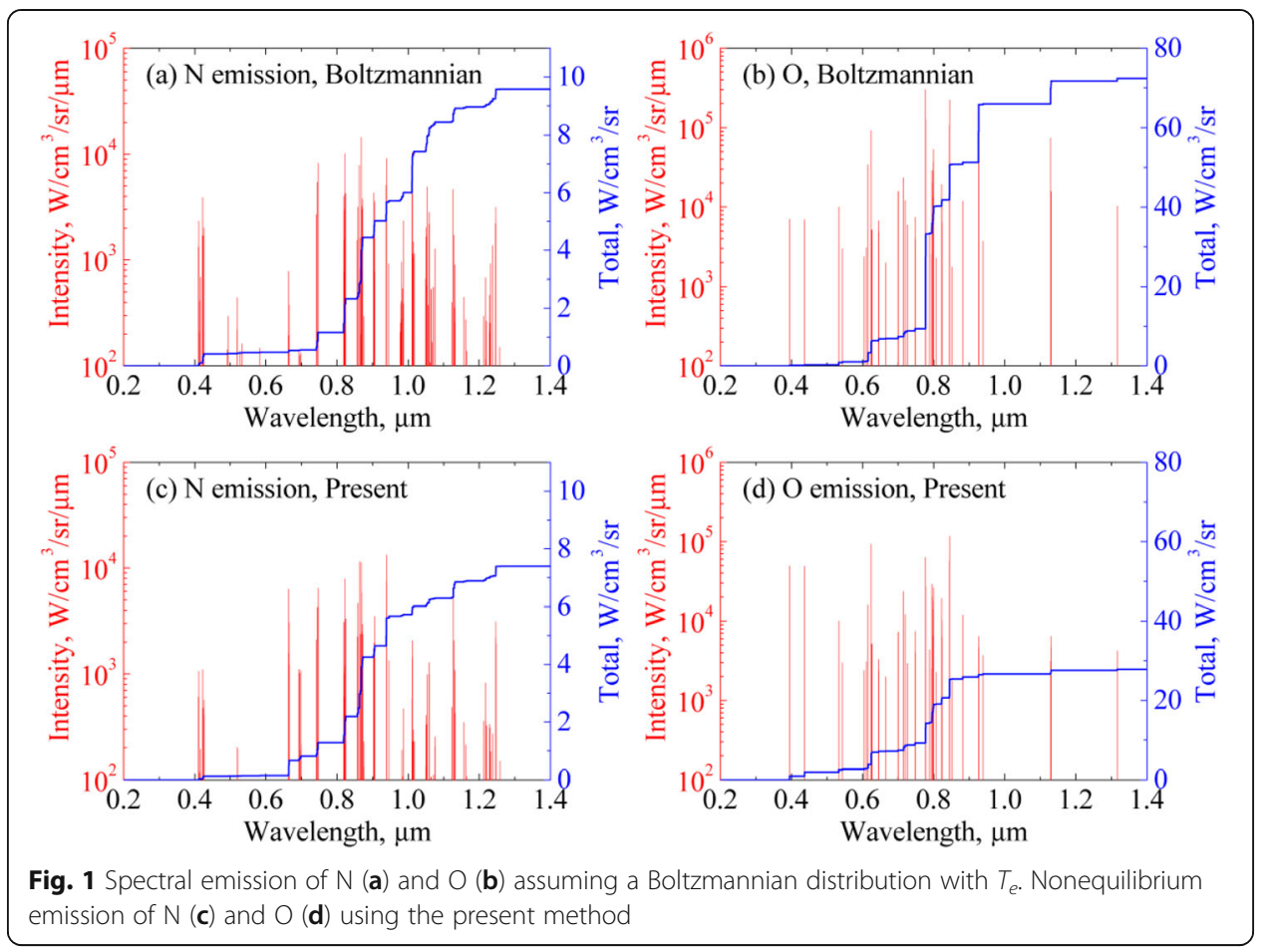

where $e, v, J$ are the quantum number of electronic, vibrational and rotational energy levels. In this case, the degeneracy of the $i$ th level is

$$
g_{i}=g_{e} \sum_{v, J} g_{J} \exp \left(-\frac{G(v)}{k T_{v}}-\frac{F(J)}{k T_{r}}\right)
$$

To investigate the difference between the nonequilibrium and equilibrium radiation of diatomic molecules, we reconsider the air plasma cell listed in Table 2. We only calculate $\mathrm{N}_{2}$ as an example. The first positive system $\left(B^{3} \Pi_{g}-A^{3} \Sigma_{u}^{+}\right)$and the second positive system $\left(C^{3} \Pi_{u}-B^{3} \Pi_{g}\right)$ of $\mathrm{N}_{2}$ are studied.

We first calculate the nonequilibrium electronic level population of $\mathrm{N}_{2}$. According to Table 3 and Eq. (4), radiative recombination coefficients can be obtained. Based on the transition probabilities in the literature [12], we substitute Eq. (2) into Eq. (3) and calculate the nonequilibrium coefficients, which are listed in Table 4 . These nonequilibrium coefficients suggest that, compared with the population determined by the SahaBoltzmann equation, the population of $B^{3} \Pi_{g}$ is 5.89 times larger, whereas the population of $C^{3} \Pi_{u}$ is 0.25 times smaller.

Table 3 The values of $\omega_{i}$ for diatomic molecules

\begin{tabular}{|c|c|c|c|c|c|c|c|c|c|c|c|c|}
\hline & \multicolumn{3}{|l|}{$\mathrm{N}_{2}$} & \multicolumn{2}{|l|}{$\mathrm{O}_{2}$} & \multicolumn{7}{|l|}{ NO } \\
\hline & $A^{3} \Sigma_{u}^{+}$ & $B^{3} \Pi_{g}$ & $C^{3} \Pi_{u}$ & $x^{3} \Sigma_{g}^{-}$ & $B^{3} \Sigma_{u}^{-}$ & $x^{2} \Pi_{r}$ & $A^{2} \Sigma^{+}$ & $B^{2} \Pi_{r}$ & $c^{2} \Pi_{r}$ & $D^{2} \Sigma^{+}$ & $B^{\prime 2} \Delta$ & $E^{2} \Sigma^{+}$ \\
\hline$l_{i} \mathrm{~cm}^{-1}$ & 75,427 & 66,012 & 36,494 & 98,401 & 48,608 & 76,800 & 32,834 & 30,867 & 24,620 & 23,715 & 16,435 & 16,171 \\
\hline$\omega_{i}$ & 1.21 & 1.29 & 1.73 & 1.06 & 1.50 & 1.20 & 1.83 & 1.89 & 2.11 & 2.15 & 2.58 & 2.61 \\
\hline
\end{tabular}


Table 4 The nonequilibrium coefficients

\begin{tabular}{ll}
\hline Levels & $\boldsymbol{b}_{\boldsymbol{i}}$ \\
\hline$B^{3} \Pi_{g}$ & 5.89 \\
$C^{3} \Pi_{u}$ & 0.25 \\
\hline
\end{tabular}

Figure 2 shows the spectral emission of $\mathrm{N}_{2}$. Here, we only consider the bound-bound transitions, and the Voigt profile is used as the lineshape. The results in Fig. 2(a) are based on the multi-temperature (multi-T) model, where the population of electronic, vibrational, and rotational energy levels is assumed to be Boltzmannian distribution characterized by $T_{e}, T_{v}$, and $T_{r}$, respectively. The results in Fig. 2(b) are based on our method. Specifically, the population of electronic levels is calculated by the abovementioned method, whereas the population of vibrational and rotational levels is assumed to be Boltzmannian distribution characterized by $T_{v}$ and $T_{r}$, respectively.

Compared with Fig. 2(b), the intensity of the first positive system in Fig. 2(a) is significantly larger, while the intensity of the second positive system is slightly smaller. The results indicate that the multi-T model overestimate the emission of $\mathrm{N}_{2}$. The overestimation is attributed to the error in the population of electronic levels.

\section{Application in hypersonic flow}

To verify the application of the present method in hypersonic nonequilibrium air plasmas, we chose to study the cell conditions listed in Ref. [13]. We did not discuss the equilibrium states, but focused the attention on the nonequilibrium AVCO R-156 experiments.

Based on the nonequilibrium cell condition in Ref. [13], we calculated the fine spectra with a high resolution $(0.001 \mathrm{~nm})$. The results are shown in Fig. 3(a). Here, the boundbound, bond-free, and free-free transitions of particles are considered. It is seen that atomic lines are dominant due to the high temperature. Regarding to the molecular bands, the emission of $\mathrm{NO}$ and $\mathrm{O}_{2}$ makes the spectral intensity below $200 \mathrm{~nm}$ relatively strong. Meanwhile, the peak intensity at $\sim 400 \mathrm{~nm}$ is mainly due to the emission of $\mathrm{N}_{2}^{+}$. However, the emission of $\mathrm{N}_{2}$ is relatively weak.

Figure 3(b) shows a comparison with the computational results of NEQAIR code and the nonequilibrium AVCO R-156 experimental spectra. The resolution of our calculated spectrum is converted to $20 \mathrm{~nm}$, which is the same as the resolution of the NEQA
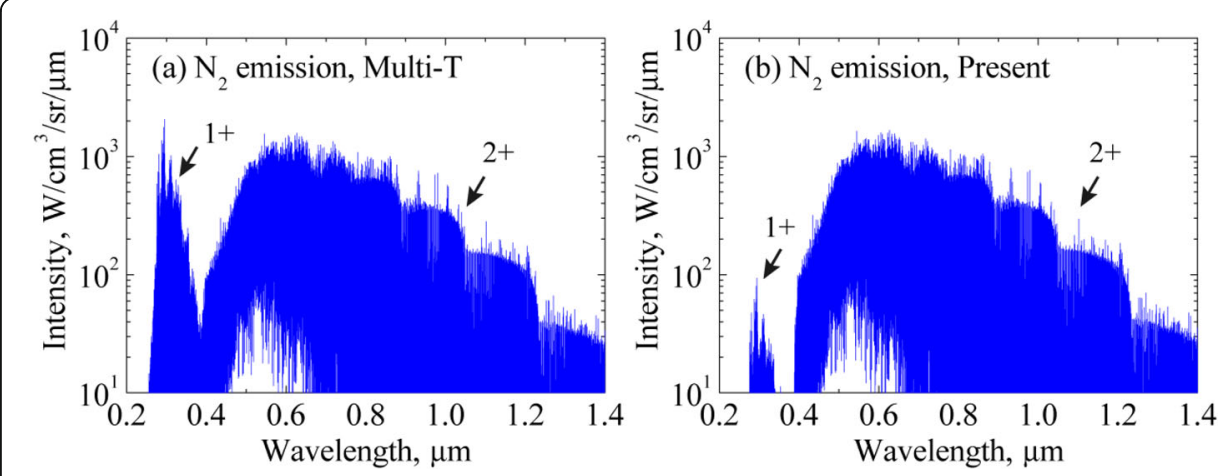

Fig. 2 Emission of $\mathrm{N}_{2}$ based on the multi-T model (a) and the present method (b) 

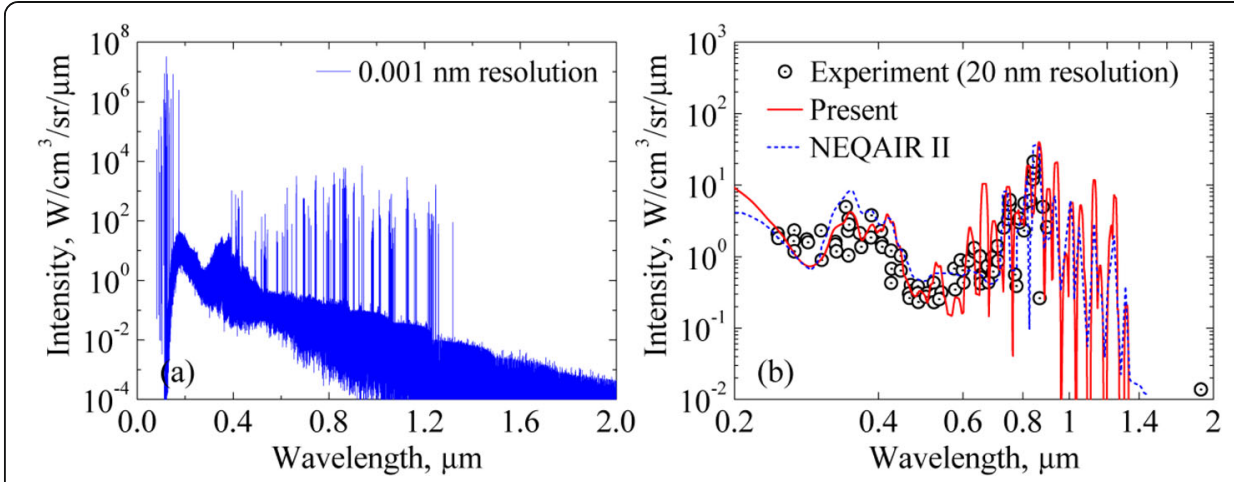

Fig. 3 Emission of the AVCO R-156 nonequilibrium air plasma. (a) The calculated fine spectra. (b) A comparison with the NEQAIR II and the measured data

IR results and the measured data. It is clearly seen that the present results are in good agreement with the NEQAIR and the measured data. This indicates that the present method is reasonable, and has a good accuracy. In the spectral region of $650-700 \mathrm{~nm}$, there is a deviation between our results and the experimental data. It can be attributed to the following two reasons. One is that the transition probabilities related to the atomic lines within $663-665 \mathrm{~nm}$ are inaccurate to a certain extent, as Table 5 shows [26]. The other is that, owing to the lack of transition probabilities, the spontaneous transitions from the upper levels to the 12th level are not included in the present calculation.

Since it is not necessary to solve the rate equations of energy level populations, the present method is simpler than most of QSS models including the NEQAIR code. It is applicable for the 11-species air plasmas that contains $\mathrm{N}, \mathrm{N}^{+}, \mathrm{O}, \mathrm{O}^{+}, \mathrm{N}_{2}, \mathrm{~N}_{2}^{+}, \mathrm{O}_{2}, \mathrm{O}_{2}^{+}$, $\mathrm{NO}, \mathrm{NO}^{+}$and free electrons. In fact, if the corresponding gas components are added, this method is also suitable for a variety of hypersonic applications.

\section{Conclusions}

We developed a simplified method for calculating spectral emission of nonequilibrium air plasmas. The method introduces nonequilibrium coefficients into the population of each energy level governed by the Saha-Boltzmann equation. By considering the three significant radiative processes, the nonequilibrium coefficients can be calculated. Thus, the nonequilibrium populations can be obtained. For diatomic molecules, an approach to the determination of the radiative recombination coefficient and the energy level degeneracy is presented. Based on the approach, we can also calculate the nonequilibrium coefficients. A typical air plasma cell is chosen to investigate the difference between the

Table 5 Transition probabilities related to atomic lines within $663-665 \mathrm{~nm}$

\begin{tabular}{lll}
\hline Wavelength, $\AA$ & $\boldsymbol{A}_{\boldsymbol{k} \text { i }} \mathbf{1 0}^{\mathbf{8}} \mathbf{s}^{\mathbf{- 1}}$ & Possible deviation \\
\hline 6644.96 & 0.0311 & $25 \%$ \\
6653.46 & 0.0244 & $25 \%$ \\
6656.51 & 0.0193 & $25 \%$ \\
6636.94 & 0.0125 & $25 \%$ \\
6646.51 & 0.0194 & $25 \%$ \\
\hline
\end{tabular}


nonequilibrium and equilibrium emission of atoms and molecules. It is suggested that the multi-T model overestimate the spectral emission in comparison with our results. Application of the present method to the nonequilibrium AVCO R-156 flowfield was shown. The results indicate that the present method agrees well with the NEQAIR code and the experimental data. This work offers a simple method to the calculation of nonequilibrium air plasma radiation, which will be useful in hypersonic engineering.

\section{Acknowledgements}

The authors would like to thank Prof. Junbo Yang and Prof. Juncai Yang for their helpful work. The authors are thankful to the reviewers for their valuable comments to improve the quality of the manuscript.

\section{Authors' contributions}

$\mathrm{XH}$ proposed the method for calculating the bound electronic energy level population of atoms and molecules with the help of TJ. CG collected the quantum data of atoms and molecules. XH performed the calculation and data analysis with the help of CG and TJ. XH and CG wrote the manuscript. TJ reviewed and processed the manuscript. All authors read and approved the final manuscript.

\section{Funding}

This work is supported by the National Numerical Windtunnel project (NNW2019ZT3-B07) and the National Natural Science Foundation of China (11674395).

\section{Availability of data and materials}

Data supporting the results of this article can be found, including data generated during the study. According to reasonable requests, the minimal datasets necessary to interpret and replicate the results of this article can be obtained from the corresponding author.

\section{Declarations}

\section{Competing interests}

The authors declare that they have no competing interests.

\section{Author details}

${ }^{1}$ Department of Physics, National University of Defense Technology, Changsha 410073, China. ${ }^{2}$ Computational Aerodynamics Institute, China Aerodynamics Research and Development Center, Mianyang 621000, China.

Received: 2 December 2020 Accepted: 17 May 2021

Published online: 22 June 2021

\section{References}

1. Surzhikov ST (2012) Radiative-collisional models in non-equilibrium aerothermodynamics of entry probes. J Heat Transf 134(3):031002. https://doi.org/10.1115/1.4005127

2. Park C (1990) Nonequilibrium hypersonic aerothermodynamics. Wiley Press, New York

3. Arnold JO, Whiting E, Lyle GC (1969) Line by line calculation of spectra from diatomic molecules and atoms assuming a Voigt line profile. J Quant Spectrosc Radiat Transf 9(6):775-798. https://doi.org/10.1016/0022-4073(69)90075-2

4. Dong SK, Ma Y, Tan HP (2008) Modeling of high-temperature air species nonequilibrium spectral radiation properties. J Thermophysics Heat Transfer 22(2):301-305

5. Shang JS, Surzhikov ST (2010) Simulating nonequilibrium flow for ablative earth reentry. J Spacecraft Rockets 47(5):806815

6. Fujita K, Sato S, Takashi AT (2002) Experimental investigation of air radiation from behind a strong shock wave. J Thermophys Heat Transf 16(1):77-82. https://doi.org/10.2514/2.6654

7. Park C (1985) Nonequilibrium air radiation (NEQAIR) program: user's manual, In: NASA Report No 1985-30780

8. Liu Y, Prabhu D, Trumble KA, Saunders D, Jenniskens P (2010) Radiation modeling for the reentry of the Stardust sample return capsule. J Spacecr Rocket 47(5):741-752. https://doi.org/10.2514/1.37813

9. Ozawa T, Modest MF, Levin DA (2010) Spectral module for photon Monte Carlo calculations in hypersonic nonequilibrium radiation. J Heat Transf 132(2):023406. https://doi.org/10.1115/1.4000242

10. Bansal A, Modest MF, Levin DA (2011) Multi-scale k-distribution model for gas mixtures in hypersonic nonequilibrium flows. J Quant Spectrosc Radiat Transf 112(12):1213-1221. https://doi.org/10.1016/j.jqsrt.2010.09.012

11. Landshoff RKM, Magee JL (1969) Thermal radiation phenomena, I: radiative properties of air. Plenum Publishing Corporation, New York

12. Laux CO (1993) Optical diagnostics and radiative emission of air plasmas. Dissertation, Stanford University

13. Moreau S, Laux CO, Chapman DR, MacCormack RW (1992) A more accurate nonequilibrium air radiation code: NEQAIR second generation, In: AIAA Paper No. 1992-2968. 23rd Plasmadynamics and Laser Conference, Nashville, TN

14. Whiting E, Park C, Liu Y, Arnold J, Paterson J (1996) NEQAIR96, nonequilibrium and equilibrium radiative transport and spectra program: user's manual, NASA/Ames Research Center, Moffett Field, CA, NASA Reference Publication 1389

15. Bansal A, Modest MF, Levin DA (2009) Correlated-k distribution method for atomic radiation in hypersonic nonequilibrium flows. In: AIAA Paper No. 2009-1027, 47th AIAA Aerospace Sciences Meeting and Exhibit, Orlando, Florida 
16. He X, Chang S-L, Dai S-A, Yang J-C (2013) Simulation of radiative transfer in nonequilibrium plasmas containing $N$ and O species based on the approximate collision-radiative method. Chin Phys Lett 30(11):114401. https://doi.org/10.1088/02 $56-307 X / 30 / 11 / 114401$

17. He X, Dang W-H, Jia H-H, Yin H-W, Zhang H-L, Chang S-L, Yang J-C (2014) Analysis of atomic electronic excitation in nonequilibrium air plasmas. Chin Phys Lett 31(9):095204. https://doi.org/10.1088/0256-307X/31/9/095204

18. Laux CO, Moreau S, Kruger C (1992) Experimental study and improved modeling of high-temperature air radiation. In: AIAA Paper No. 1992-2969, 23rd Plasmadynamics and Laser Conference, Nashville, TN

19. Park C (1985) Calculation of nonequilibrium radiation in the flight regimes of aeroassisted orbital transfer vehicles. Thermal Design Aeroassisted Orbital Transfer Vehicles 96:395-418

20. Park C (1993) Review of chemical-kinetic problems of future NASA mission, I: earth entries. J Spacecr Rocket 7(3):385398

21. Tauber ME, Palmer GE, Yang L (1992) Earth atmospheric entry studies for manned Mars mission. J Thermophys Heat Transf 6(2):193-199. https://doi.org/10.2514/3.345

22. Shang JS, Surzhikov ST (2011) Simulating Stardust earth reentry with radiation heat transfer. J Spacecraft and Rockets 48(3):385-396

23. Lamet JM, Babou Y, Rivière P, Perrin MY, Soufiani A (2008) Radiative transfer in gases under thermal and chemical nonequilibrium conditions: application to earth atmospheric re-entry. J Quant Spectrosc Radiat Transf 109(2):235-244. https://doi.org/10.1016/j.jgsrt.2007.08.026

24. Peach G (1970) Continuous absorption coefficients for non-hydrogenic atoms. Memoirs of the Royal Astronomical Society $73: 1-123$

25. Herzberg G (1983) Molecular spectra and molecular structure, I: spectra of diatomic molecules. Science Press, Beijing

26. Wiese WL, Smith MW, Glennon BM (1966) Atomic transition probabilities, I: hydrogen through neon. National Standard Reference Data Series, National Bureau of standards 4

\section{Publisher's Note}

Springer Nature remains neutral with regard to jurisdictional claims in published maps and institutional affiliations.

\section{Submit your manuscript to a SpringerOpen ${ }^{\circ}$ journal and benefit from:}

- Convenient online submission

- Rigorous peer review

- Open access: articles freely available online

- High visibility within the field

- Retaining the copyright to your article

Submit your next manuscript at $>$ springeropen.com 